\title{
Vector Analysis of High Astigmatism ( $\geq 2.0$ Diopters) Correction after Small-Incision Lenticule Extraction with Stringent Head Positioning and Femtosecond Laser-Assisted Laser in Situ Keratomileusis with Compensation of Cyclotorsion
}

Jihong Zhou

Beijing Tongren Eye Center, Beijing Tongren Hospital, Capital Medical University

Wei Gu

Beijing Aier-Intech Eye Hospital

\section{Yan Gao}

Beijing Aier-Intech Eye Hospital

Guoli He

Beijing Aier-Intech Eye Hospital

Fengju Zhang ( $\sim$ zhangfj126@126.com)

Beijing Tongren Eye Center, Beijing Tongren Hospital, Capital Medical University

\section{Research Article}

Keywords: femtosecond laser-assisted laser in situ keratomileusis, small-incision lenticule extraction, astigmatism, compensation of cyclotorsion, stringent head positioning

Posted Date: October 22nd, 2021

DOI: https://doi.org/10.21203/rs.3.rs-929383/v1

License: (c) (i) This work is licensed under a Creative Commons Attribution 4.0 International License. Read Full License

Version of Record: A version of this preprint was published at BMC Ophthalmology on April 5th, 2022. See the published version at https://doi.org/10.1186/s12886-022-02384-0. 


\section{Abstract}

PURPOSEX To compare the astigmatic correction by vector analysis in the high myopic astigmatism between femtosecond laser-assisted laser in situ keratomileusis with compensation of cyclotorsion (FSLASIK) and small-incision lenticule extraction(SMILE) with stringent head positioning.

SETTING: Beijing Aier-Intech Eye Hospital, Beijing, China.

DESIGN: Retrospective case series.

METHOD: Patients who had correction of myopic astigmatism of 2 diopters (D) or more had either FSLASIK with compensation of cyclotorsion or SMILE with stringently control the head position were included. Results of vision and refraction were analyzed and compared between groups with the right eye.

RESULT: The study enrolled 94 patients (41eye in the FS-LASIK with compensation of cyclotorsion and 53 eyes in SMILE with stringently control the head position). The mean preoperative manifest cylinder was $-2.65 \pm 0.77 \mathrm{D}$ in FS-LASIK and 2.51 $\pm 0.56 \mathrm{D}$ in SMILE group. ( $P=0.302)$. At 12-month, there was no significant difference between the two groups in uncorrected distance visual acuity (UDVA, $P=0.274$ ) and postoperative spherical equivalent $(P=0.107) .46 .3 \%$ and $24.5 \%$ of eyes were within $0.25 \mathrm{D}$ post-op cylinder, $78 \%$ and $66 \%$ of eyes were within $0.5 \mathrm{D}$ post-op cylinder for FS-LASIK group and SMILE group, respectively $(P=0.027, P=0.202)$. Vector analysis showed comparable target-induced astigmatism ( $T I A$, $P=0.114)$, surgically induced astigmatism vector $(S I A, P=0.057)$, difference vector ( $D V, P=0.069)$ and the angle of error ( $A E, P=0.213$ ) between groups. Index of success (IOS) was 0.18 in the FS-LASIK and 0.24 in the SMILE $(P=0.024)$, and significant difference for two groups.

CONCLUSION: FS-LASIK with compensation of cyclotorsion had favorable correction in high myopic astigmatism ( $\geq 2.0$ diopters) compared to SMILE with stringent head positioning at 12-month.

\section{Introduction}

Femtosecond laser-assisted laser in situ keratomileuses (FS-LASIK) was first reported by Ratkay-Traub I et al. in 2003.[1] This procedure has many superiorities over the mechanical microkeratome, fewer side effects as free caps, irregular flaps, and buttonholes et al., while complicated with the femtosecond laser making the flap then the excimer laser performing the corneal reshaping.

Small-incision femtosecond lenticule extraction (SMILE, Carl Zeiss Meditec AG) has obtained popularity in recent years as another new paradigm for myopic refractive errors. Sekundo W et al. [2] carried out the first clinical report in 2008. The SMILE procedure is flapless, reserved more nerve fibers and corneal biomechanical strength; thus, dry eye and complications correlated with flap diminished potentially compared with FS-LASIK. Many studies have proved the two groups' comparable visual and refractive outcoming, safety, efficacy, and predictability. $[3,4]$ The precision of astigmatism correction in the SMILE 
platform has been suspect because of the lack of cyclotorsion control. A trend toward undercorrection and misalignment with SMILE in 6 months follow-up was reported by Khalifa MA et al. [5] Chan TCY et al. [6] showed SMILE procedure was less efficacious correction with low to moderate myopic astigmatism but comparable to high astigmatism ( $\geq 3.0 \mathrm{D}$ ) correction with FS-LASIK and followed up only three months.

The aim of the retrospective study used vector analysis to evaluate the efficacy of astigmatic correction in high myopic astigmatism ( $\geq 2.0$ diopters) between FS-LASIK with compensation of cyclotorsion and SMILE with a stringent head positioning for long-term (12-month) follow-up.

\section{Patients And Methods}

This study was patients who had FS-LASIK or SMILE between September 2013 and June 2020 by the same surgery (J.H. Zhou). Patients with myopic astigmatism of 2.0D or more were involved analysis. All data for the study were collected and analyzed by the Institutional Review Board (IRB), Beijing Aierlntech Eye Hospital, Beijing, China. The study protocol was approved by the ethics committee and adhered to the Declaration of Helsinki's tenets. All patients were given information about the risks and benefits of the procedures and signed informed consent before surgery.

All patients had a comprehensive ophthalmic examination and exclude eye disease besides myopia and myopic astigmatism. Exclusion criteria were suspicion of keratoconus on corneal topography, ocular inflammation, and infection.

The preoperative evaluation contained uncorrected distance visual acuity (UDVA), corrected distance visual acuity (CDVA), intraocular pressure (IOP) by noncontact tonometer (Nidek NT-510; Japan), slit-lamp biomicroscopy, subjective manifest and cycloplegic refraction by a comprehensive optometry station (Nidek AOS-1500; Japan), corneal topography and wavefront aberration with an OPD Scan III (NIDEK, Technologies, Gamagori, Japan ), cornea thickness scanning with a PENTECAM (Oculus Optikgeräte $\mathrm{GmbH}$ ). Follow-up visits were scheduled at $1 \mathrm{~d}, 7 \mathrm{~d}, 1 \mathrm{~m}, 3 \mathrm{~m}, 6 \mathrm{~m}$, and 12-month regularly. Postoperatively, refractions were recorded by an automatic refractometer (NIDEK ARK-510, Japan); UDVA, CDVA, IOP, topography, and wavefront aberration were recorded during follow-ups.

Before surgery, a topical anesthetic (benoxinate hydrochloride $0.4 \%$ ) was instilled two or three times in the conjunctival fornix of the eye.

FS-LASIK procedure was performed using the FS200 (WaveLight ${ }^{\circledR}$ AlconSurgical, Fort Worth, TX, USA) femtosecond laser to create the flap (100-110 $\mu \mathrm{m}$ thickness, 8.5- $8.7 \mathrm{~mm}$ diameter, and 40-degree superior hinge), followed by the EX-500 excimer laser ablation with pupil-tracking and compensation of cyclotorsion by iris registration; the diameter of the optical zone was employed for 6.00 to $6.7 \mathrm{~mm}$. After the ablation, the corneal flap was irrigated with a saline solution and repositioned in the stromal bed. 
SMILE procedure was performed using the VisuMax femtosecond laser system (Carl Zeiss Meditec AG, Jena, Germany) with a $500 \mathrm{kHz}$ repetition rate. We used the small (S) curved interface cone in all cases. In all processes, the surgeon made manual limbal markings at the $0^{\circ}$ to $180^{\circ}$ axis in front of the slit-lamp preoperatively [7] (Figure 1). We appended the red markings on the operation bed and the red line on the slit lamps (Figure 2). The surgical assistant makes the edge of the patient's earlobes correspond to the red markings on the operating bed. Then, the surgeon makes the patient's inner and outer canthal angle parallel to the red line to adjust head cyclotorsion. Finally, we regulated the limbal markings to match the horizontal line of the microscope cross, and suction was applied. The following parameters were applied: cut energy, 105 to $135 \mathrm{~nJ}$; the cap and lenticular spot-track-distance, $4.5 \mu \mathrm{m}$; the cap side and lenticular side spot-track-distance 1.8 to $2.0 \mu \mathrm{m}$; the cap thickness, 100 to $130 \mu \mathrm{m}$, cap diameter, 7.1 to $7.8 \mathrm{~mm}$; lenticule diameter, 6.0 to $6.7 \mathrm{~mm}$, depending on the preoperative corneal thickness, pupil size and the refractive error to be corrected. The small incision was created at the 10 o'clock position with a two mm side cut. The lenticular was gently separated with a spatula and extracted with a pair of forceps.

\section{Vector Analysis}

We carried out the vector analysis for astigmatic correction depending on the Alpins method,[8] three fundamental vectors were examined.

Q Target-induced astigmatism (TIA) vector is the vector of the astigmatic correction that the surgery was attempted.

4 The surgically induced astigmatism (SIA) vector is interpreted as the astigmatic achieved by the surgery actually caused.

૫ The difference vector (DV) was defined as the vectorial difference between the TIA and SIA vectors.

We could compare outcomes of astigmatism correction from above the three vectors (TIA, SIA, DV) of various relationships.

૧ The magnitude of error (ME) is the arithmetic difference between the SIA and TIA.

4 The angle of error (AE) is the angle described by the vector of SIA versus TIA. AE is positive if the achieved correction is on an axis counterclockwise (CCW) to where it was intended and negative if the achieved correction is clockwise (CW) to its intended axis. The absolute angle of error is the angle between the axis of the SIA and TIA.[9]

4. Correction index $(\mathrm{CI})$ is defined as the SIA divided by the TIA. The value might be one preferably, and astigmatism was under correction if $\mathrm{Cl}$ was lower than 1 .

[ Index of success (IOS) is the DV divided by the TIA. The vale should be 0 , preferably.

\section{STATISTICAL ANALYSIS}


Only the right eyes were analyzed to prevent relevance between two eyes. Data are presented as mean \pm standard deviation (SD). An independent t-test was used to estimate continuous variables between the two groups. Linear regression analyses were performed for TIA and SIA (or attempted and achieved sphere). Analysis of covariance (ANCOVA) was used to adjust for different preexisting pre-sphere of the baseline. Categorical variables were assessed using Pearson's Chi-squared test. A repeated-measures ANOVA was applied to compare variables among each follow-up. The data were plotted in sets of 9 standard graphs that comprised efficacy, predictability, safety, and stability using Microsoft Excel templates designed by the London Vision Clinic. ${ }^{10}$ Vector analyses applied AstigMATIC software. [11] A pvalue less than 0.05 was considered statistically significant.

\section{Results}

94 eyes of 94 patients were enrolled. 41 eyes were in the FS-LASIK group, and 53 eyes were in the SMILE group. The mean age was $27.59 \pm 7.47$ and $29.79 \pm 7.15$ years in the FS-LASIK group and SMILE group, respectively $(P=0.149)$. Table 1 shows the baseline characteristics of the two groups. The mean of the preoperative manifest cylinder was $-2.65 \pm 0.77$ (range from -2.00 to $-5.75 \mathrm{D}$ ) in the FS-LASIK group and $-2.51 \pm 0.56$ (range from -2.00 to $-4.50 \mathrm{D}$ in the SMILE group $(P=0.302)$. Preoperatively, there were no statistically significant difference in pre-CDVA $(P=0.144)$, sex rates $(P=0.104)$, central corneal thickness $(P=0.729)$, age $(P=0.149)$ and IOP $(P=0.738)$ while statistically significant differences in the manifest sphere $(P=0.044)$ and the spherical equivalent (SEQ) $(P=0.027)$ between the two groups. No intraoperative complications occurred in all surgeries. 
Table 1

Preoperative characteristics of eyes in FS-LASIK group and SMILE group

\begin{tabular}{|llll|}
\hline Parameter & FS-LASIK (41Eyes) & SMILE (53 Eyes) & $P$ \\
\hline CDVA-logMAR (Mean \pm SD) & $0.026 \pm 0.04$ & $0.013 \pm 0.04$ & 0.144 \\
\hline Sphere (Mean \pm SD) & $-5.58 \pm 2.88$ & $-4.50 \pm 1.96$ & 0.044 \\
\hline Cylinder (Mean \pm SD) & $-2.65 \pm 0.77$ & $-2.51 \pm 0.56$ & 0.302 \\
\hline SEQ (Mean \pm SD) & $-6.90 \pm 2.76$ & $-5.75 \pm 1.94$ & 0.027 \\
\hline CCT (Mean $\pm S D)$ & $542.66 \pm 29.33$ & $544.60 \pm 24.91$ & 0.729 \\
\hline Sex (Male: N, \%) & $14,20.6$ & $26,32.5$ & 0.104 \\
\hline Age (Mean $\pm S D$ & $27.59 \pm 7.47$ & $29.79 \pm 7.15$ & 0.149 \\
\hline IOP (Mean $\pm S D)$ & $16.00 \pm 2.49$ & $15.83 \pm 2.39$ & 0.738 \\
\hline $\begin{array}{l}\text { CDVA= corrected distance visual acuity; logMAR= logarithm of the minimum angle of resolution; } \\
\text { CCT= central corneal thickness; IOP=Intraocular pressure; SEQ=spherical equivalent }\end{array}$ \\
\hline $\begin{array}{l}\text { FS-LASIK=femtosecond laser-assisted laser in situ keratomileusis; SMILE= small-Incision lenticule } \\
\text { extraction. }\end{array}$
\end{tabular}

\section{Efficacy and Safety}

Table 2 showed the postoperative characteristic of eyes in FS-LASIK and SMILE. Postoperatively at 12 months, we found no significant difference in the UDVA ( $P=0.274), C D V A(P=0.51)$, efficacy index $(P=0.828)$, and safety index ( $P=0.285)$; Figure 3A showed 40 eyes (98\%) in FS-LASIK and 49 eyes $(92 \%)$ in SMILE had a UDVA of $20 / 20$ or better $(P=0.274)$. Figure 3B revealed $95.1 \%$ (49 eyes) in the FS-LASIK group and $92.5 \%$ (49 eyes) in the SMILE group had postop UDVA the same or better than preop CDVA $(P=0.600)$. No eye in FS-LASIK or SMILE group lost one or more lines in post CDVA. (Figure 3C) The corneal complication was not detected in any patient postoperatively.

Table 2

Postoperative characteristics of eyes at 12 months after FS-LASIK and SMILE 
FS-LASIK (41Eyes)

\begin{tabular}{|c|c|c|c|}
\hline Parameter & Mean \pm SD & Mean \pm SD & $P^{*}$ \\
\hline UDVA (logMAR) & $-0.03 \pm 0.10$ & $-0.05 \pm 0.08$ & 0.274 \\
\hline CDVA (logMAR) & $-0.03 \pm 0.09$ & $-0.04 \pm 0.08$ & 0.510 \\
\hline Efficacy Index & $1.16 \pm 0.24$ & $1.17 \pm 0.21$ & 0.828 \\
\hline Safety Index & $1.19 \pm 0.19$ & $1.14 \pm 0.26$ & 0.285 \\
\hline Sphere & $-0.08 \pm 0.60$ & $0.18 \pm 0.42$ & 0.028 \\
\hline Cylinder & $-0.46 \pm 0.32$ & $-0.57 \pm 0.40$ & 0.205 \\
\hline SEQ & $-0.31 \pm 0.63$ & $-0.10 \pm 0.49$ & 0.107 \\
\hline Attempted & $-6.90 \pm 2.77$ & $-4.50 \pm 1.96$ & 0.114 \\
\hline Achieved & $-6.58 \pm 2.77$ & $-4.68 \pm 1.96$ & 0.491 \\
\hline \multicolumn{4}{|c|}{$\begin{array}{l}\text { *: Analysis of covariance, pre-sphere was used to adjust for preexisting differences of the baseline. } \\
\text { CDVA= corrected distance visual acuity; logMAR= logarithm of the minimum angle of resolution; } \\
\text { UDVA= uncorrected distance visual acuity; SEQ=spherical equivalent, FS-LASIK= femtosecond laser- } \\
\text { assisted laser in situ keratomileusis; SMILE= small-Incision lenticule extraction. }\end{array}$} \\
\hline \multicolumn{4}{|c|}{ Predictability } \\
\hline
\end{tabular}

There was no significant difference in the SEQ $(P=0.107)$ and cylinder $(P=0.205)$, but significant difference in sphere $(P=0.028)$ at 12 months. Figure $4 A$ shows a scatter plot of attempted versus achieved SEQ refraction for the FS-LASIK and SMILE groups. Within $\pm 0.50 \mathrm{D}$ of emmetropia, 27 eyes (65.9\%) after FS-LASIK were lower than 45 eyes (84.9\%) after SMILE ( $P=0.031)$; 36eyes (87.8\%) for FSLASIK group and 51eyes (94.4\%) for SMILE group were comparable within $\pm 1.00 \mathrm{D}$ ( $P=0.248$; Figure 4B). There is no statistically significant difference in spherical equivalent (SE) in FS-LASIK and SMILE throughout the 1 to 12 months postoperative period ( $P=0.502$; Figure $4 \mathrm{C}$ ).

\section{Vector Analysis}

Table 3 shows a comparative analysis of the vector astigmatic results. Figure $5 \mathrm{~A}$ displayed amplitude of astigmatism: post-op and pre-op, 19 eyes (46.3\%) in FS-LASIK and 13 eyes (24.5\%) in SMILE were less than or equal to $0.25 \mathrm{D}$ cylinder $(\mathrm{P}=0.027)$; correspondingly, 32 eyes $(78 \%)$ and 35 eyes $(66 \%)$ had a postoperative cylinder $\leq 0.50 \mathrm{D}(\mathrm{P}=0.202)$, and 38 eyes $(92.7 \%)$ and 48 eyes $(90.6 \%)$ were $\leq 1.00 \mathrm{D}$ $(P=0.715)$. Figure $5 B$ presented a scatter plot of TIA versus SIA at 12 -months. Figure $5 \mathrm{C}$ showed astigmatism of the angle of error $(A E)$ between the two procedures $(P=0.231)$. Figure 6 shows singleangle polar plots with a vector mean of TIA, SIA, DV, and CI for FS-LASIK (A) and SMILE (B) at 12 months. There was no significant difference in TIA $(P=0.114)$, SIA $(P=0.057)$, the magnitude of error $(M E$, $P=0.425)$, difference vector ( $D V, P=0.069), C l(P=0.232)$ between FS-LASIK and SMILE; except for index of success (IOS) was significantly higher in SMILE than FS-LASIK $(P=0.024)$. 
Table 3

Vector analysis results of astigmatic correction at 12 months after FS-LASIK and SMILE 
FS-LASIK (41)

SMILE (53)

\begin{tabular}{|c|c|c|c|}
\hline Parameter & Mean \pm SD & Mean \pm SD & $P^{*}$ \\
\hline $\mathrm{TIA}(\mathrm{D})$ arithmetic mean & $2.65 \pm 0.77$ & $2.51 \pm 0.56$ & 0.114 \\
\hline Vector mean & 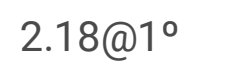 & 2.24@2º & \\
\hline TIAx & $-2.18 \pm 1.52$ & $-2.24 \pm 0.96$ & 0.876 \\
\hline TIAy & $-0.09 \pm 0.78$ & $-0.15 \pm 0.83$ & 0.763 \\
\hline SIA (D) arithmetic mean & $2.43 \pm 0.73$ & $2.18 \pm 0.68$ & 0.057 \\
\hline Vector mean & 1.95@2º & 1.95@0 & \\
\hline SIAx & $-1.95 \pm 1.42$ & $-1.95 \pm 0.92$ & 0.926 \\
\hline SIAy & $-0.12 \pm 0.82$ & $-0.02 \pm 0.76$ & 0.536 \\
\hline ME (D) & $-0.22 \pm 0.38$ & $-0.33 \pm 0.44$ & 0.425 \\
\hline DV (D) arithmetic mean & $0.46 \pm 0.32$ & $0.59 \pm 0.35$ & 0.069 \\
\hline Vector mean & $0.24 @ 176^{\circ}$ & 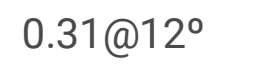 & \\
\hline DV-x & $-0.24 \pm 0.38$ & $-0.29 \pm 0.48$ & 0.842 \\
\hline DV-y & $0.03 \pm 0.34$ & $-0.13 \pm 0.39$ & 0.051 \\
\hline $\mathrm{Cl}$ & $0.92 \pm 0.14$ & $0.87 \pm 0.19$ & 0.232 \\
\hline $\mathrm{AE}$ (degree) & $2.14 \pm 12.05$ & $-1.13 \pm 11.18$ & 0.213 \\
\hline$A E_{A B S}$ & $7.76 \pm 9.40$ & $8.11 \pm 7.70$ & 0.931 \\
\hline IOS & $0.18 \pm 0.13$ & $0.24 \pm 0.13$ & 0.024 \\
\hline
\end{tabular}

*: Analysis of covariance, pre-sphere was used to adjust for preexisting differences of the baseline.

TIA: Target induced astigmatism vector

SIA: Surgically induced astigmatism vector

ME: Magnitude of error, the arithmetic difference between the magnitudes of the SIA and TIA.

Cl: Correction Index (SIA/TIA)

$\mathrm{AE}$ : Angle of error

$A E_{A B S:}$ Absolut angle of error

DV: Difference Vector

IOS: Index of success (DV/TIA)

FS-LASIK= femtosecond laser-assisted laser in situ keratomileusis; 
SMILE= small-Incision lenticule extraction.

\section{Discussion}

This study proved that SMILE and FS-LASIK had comparable efficacy and safety and in correcting high myopic astigmatism. UDVA of $20 / 25$ or better was $98 \%$ in SMILE and $100 \%$ in FS-LASIK, and CDVA was no loss in any eye for both groups. Chan T.C.Y. et al. [9] described no difference in outcomes of vision and refraction after FS-LASIK and SMILE for 3months, and Liu M. et al.'s [12] comparative study gave the similar result. Our current study showed the predictability within \pm 0.50 was $85 \%$ and $66 \%$ of spherical equivalent (SE) after SMILE and FS-LASIK ( $P=0.031)$, respectively, similar to Han T et al. [13] The predictability of SE after SMILE was better than FS-LASIK, which myopia was additional higher for FSLASIK. The stability of postoperative spherical equivalent (SE) was no significant difference, and shift forward myopia was fewer at 12-months for FS-LASIK and SMILE, paralleled previous results. $[13,14]$

We assessed astigmatic correction ( $\geq 2.0$ diopters) by using the Alpins method,[8] showed TIA, SIA, the magnitude of error (ME), difference vector (DV), correction index (CI), and angle of error (AE) had no statistically significant differences, other than the index of success (IOS), between FS-LASIK and SMILE group at 12-month follow-up. To the best of our knowledge, this study was the first study comparing a long-term result (12 months) including patients with high myopic astigmatism ( $\geq 2.0$ diopters) by vector analysis between FS-LASIK (with compensation of cyclotorsion by iris-registration) and SMILE (with a stringent head positioning and manual limbal marking to correct).

In our results, mean preoperative cylinder of -2.65D, TIA of 2.65D in FS-LASIK, -2.51 D, and TIA of $2.51 \mathrm{D}$ in the SMILE group were estimated. Postoperatively undercorrection of astigmatism was observed with a mean cylinder of -0.46 and $-0.57 \mathrm{D}$, the magnitude of error (ME) of -0.22 and $-0.33 \mathrm{D}$, correction index (Cl) of 0.92 and 0.87 in FS-LASIK and SMILE at 12-month respectively. Zhang et al. [15] reported that their correction index was 0.94 in wavefront-guided FS-LASIK and 0.88 in SMILE by the analysis for a mean preoperative cylinder of TIA 2.48 to $2.65 \mathrm{D}$ at three months. Previous results showed a certain extent undercorrection after both surgical procedures. $[6,9,15,16]$

The axis of the cylinder aligned was critical during the treatment. Ganesh et al. [17] accomplished manual cyclotorsion compensation directed by preoperative limbal marking, and also applied a 10\% overcorrection nomogram. They had a mean preoperative cylinder of $-2.48 \mathrm{D}$ and TIA of $2.19 \mathrm{D}$, then the postoperative cylinder of $-0.31 \mathrm{D}$, the magnitude of error for $-0.149 \mathrm{D}$, correction index of 0.93 , and index of success of 0.14 at three months. Pedersen IB et al. [18] suggested that a nomogram adjustment by $10 \%$ in the magnitude of astigmatism correction could be beneficial. Our study only applied about a $10 \%$ overcorrection nomogram for sphere, not for cylinder in SMILE, and long-term follow-up might be the extra favorite undercorrection. Myopic ablation was related to corneal epithelial remodeling and increased after FS-LASIK at 1-month and up to 1-year.[19] Our results were consistent with previous that high 
astigmatic correction by FS-LASIK seemed better than SMILE, but no statistically significant difference in difference vector (DV), magnitude of error (ME) and Correction index (Cl).

12-month post-operation in our result, an average difference of vector (DV) was 0.24@176 in FS-LASIK and 0.31@12 in SMILE. The average AE value was positive (2.14) after FS-LASIK and negative (-1.13) after SMILE. The absolute AE reported in the current study was 7.76 degrees in FS-LASIK and 8.11 degrees in SMILE, comparable to Pedersen et al. [18] reported AE 0.34 and absolute mean AE 8.94. Zhang JM et al. [20] showed corresponding results of AE -3.04 and absolute mean AE 6.08 after SMILE at 12month follow-up. The mean absolute AE value was slightly higher after SMILE than after FS-LASIK, but our results showed no significant difference. When calculated by vector analysis, the proportion of loss of flattening effect (FE) is $1.5 \%$ when treatment is 5 degrees misaligned, $13.4 \%$ when 15 degrees, $50 \%$ when 30 degrees et al. [21] The residual cylinder of $0.25 \mathrm{D}$ or less was $46.3 \%$ of eyes after FS-LASIK, and $24.5 \%$ of eyes after SMILE in the high astigmatic correction $(\geq 2.0 \mathrm{D})(P=0.027)$ for 12-month in ours study. In Kanellopoulos's study,[22] $82 \%$ of eyes in LASIK and $50 \%$ of eyes in SMILE had a residual cylinder of 0.25 $D$ or less for 3-month follow-up with correction of astigmatism ( $\geq 1.5 \mathrm{D})$. The difference might attribute to the disparity of astigmatic degree and shorter follow-up time. FS-LASIK by compensation of cyclotorsion expressed the better predictability of astigmatic correction.

In this study, three steps to control the compensation of cyclotorsion and others in the SMILE procedure were performed: preoperative marking on the limbal, a stringent head positioning, and limbal marking matching the horizontal line of the microscope cross, but did not apply gently rotating the cone on the eye when started suction, as the manual limbal marking method might introduce varying degrees from 3.8 to 6.0 degrees inherently.[23] Prickett $A L$ et al. observed that most of the rotations previously attributed to torsional components were probably due to non-cyclotorsion components, such as postural misalignments.[24] Chan T.C.Y et al. also emphasized the position of the head to both eyes align along an imagined horizontal line without the manual limbal marking,[9] further reason might be $86 \%$ of eyes for high astigmatism within 5 degrees or less cyclotorsion in the survey by Ganesh et al.[17] Manual limbal marking and stringent head positioning both affirmed a safe, feasible, and effective strategy to perfect the results of high astigmatic correction with SMILE.

Shen EP et al.'s [7] compared manual limbal markings versus iris-registration systems in LASIK and concluded manual limbal marking was a safe alternative when automated systems are not available. Zhao XH et al. [16] also showed that wavefront-guided FS-LASIK and optimized SMILE achieved similar outcomes for astigmatism correction. Though various methods aligned cyclotorsion for SMILE procure $[16,17,25]$ to improve astigmatism correction. In contrast, the system of iris-registration for the Visumax platform is warranted in the future to enhance its capability in astigmatic correction.

We got less efficacy in correcting astigmatism after SMILE by a stringent head positioning than FS-LASIK by an iris-registration system with compensation of cyclotorsion. An IOS of 0.00 indicates complete success in astigmatism treatment, a result of 1.00 shows no improvement on the preoperative status, and greater than 1.00 shows a deterioration in astigmatism.[8] Index of success (IOS) was various from 0.07 
to 0.17 of FS-LASIK and 0.09 to 0.15 of SMILE for 3 to 6 months follow-up in the previous result. $[9,15]$ Our study showed IOS was 0.18 of FS-LASIK and 0.24 of SMILE, with a significant difference between the two groups at 12 months. The IOS was higher than the previous might be long-term follow-up in our report. Ivarsen A et al. [26] also demonstrated a tendency for greater under-correction over time with higher degrees of astigmatic correction. Higher undercorrection with high astigmatic correction over time might be associate with corneal epithelial remodeling. [19]

The limitation of this study was its small sample size of high astigmatic correction. Varma R et al. [27] showed the prevalence of astigmatism was only 3.7\% (3.1\%-4.3\%) for high astigmatism (defined as over $2.25 \mathrm{D}$ ) in adults of Chinese Americans. From the beginning of the study, we enrolled 840 right eyes of 840 patients for high astigmatism ( $\geq 2.0$ diopters); only 94 eyes $(11.2 \%)$ finished the process at 12-month follow-up.

Second, we got postoperative refraction using an automatic optometer which might be impacted by the eye accommodation for troublesome to acquire the manifested and cycloplegic refraction at each followup. Because of the age enrolled in our study (adult), the accommodation might less affect the results. Pesudovs K [28] proved excellent agreement between autorefraction and subjective refraction, and autorefraction is a valid outcome measure of refractive surgery.

Third, the current study was retrospective design; thus, the potential selection bias could not be excluded in a random clinical trial. Even more prospective, randomized clinical trials should therefore be advanced with more sample size.

In conclusion, SMILE with stringent head positioning provided fewer favorable high astigmatic corrections ( $\geq 2.0$ diopters) at 12-months than FS-LASIK with compensation of cyclotorsion. Further study should improve SMILE with an eye-tracking system and cyclotorsion compensation to correct astigmatism accurately in the future.

\section{Declarations}

\section{Ethics approval and consent to participate}

The study protocol was a retrospective comparative study and approval by the ethics committee, Beijing AierIntech Eye Hospital (BJAIER2021IRB05). All participants were informed and consented (written) to participate before surgery.

\section{Consent for publication}

Not applicable

\section{Availability of data and material}


The datasets used and/or analyzed during the current study are available from the corresponding author on reasonable request.

\section{Competing interests:}

The authors report no conflicts of interest.

\section{Funding}

This study was supported by Research and Transformation Application of Capital Clinical Diagnosis and Treatment Technology by Beijing Municipal Commission of Science and Technology. (Z201100005520043)

\section{Authors' contributions}

$\mathrm{JHZ}$ designed and wrote the main manuscript text and prepared figures and was a major contributor in writing the manuscript. FJZ designed the work and substantively revised the manuscript text, and she is the fund applicant and supports our research. WG participated in the design and assisted with implementation. YG and GLH collected and disposed of data. All authors read and approved the final manuscript.

\section{Acknowledgements}

My deepest gratitude goes first and foremost to Professor Fengju Zhang, my supervisor, for her constant encouragement and guidance. She has walked me through all the stages of the writing of this thesis. Without her consistent and illuminating instruction, this thesis could not have reached its present form.

Second, I would like to express my heartfelt gratitude to Shaowei Li, MD, Ph.D., who greatly supported me. I am also greatly indebted to the professors and teachers at Tongren Eye Center, Tongren Hospital, Capital Medical University, and colleagues at Beijing Aierlntech Eye Hospital. They have instructed and helped me a lot in the past two years.

Last, my thanks would go to my beloved family for their loving considerations and great confidence in me all through these years. I also owe my sincere gratitude to my friends and classmates who gave me their help and time in listening to me and helping me work out my problems during the challenging course of the thesis.

\section{References}


1. Ratkay-Traub I, Ferincz IE, Juhasz T, et al. First clinical results with the femtosecond neodyniumglass laser in refractive surgery. Journal of refractive surgery. 2003; 19(2):94-99.

2. Sekundo W, Kunert K, Russmann C, et al. First efficacy and safety study of femtosecond lenticule extraction for the correction of myopia: six-month results. J Cataract Refract Surg. 2008; 34(9):1513-1520.

3. Kataoka T, Nishida T, Murata A, et al. Control-matched comparison of refractive and visual outcomes between small incision lenticule extraction and femtosecond laser-assisted LASIK. Clin Ophthalmol. $2018 ; 12: 865-873$.

4. Pietila J, Huhtala A, Makinen P, et al. Uncorrected visual acuity, postoperative astigmatism, and dry eye symptoms are major determinants of patient satisfaction: a comparative, real-life study of femtosecond laser in situ keratomileusis and small incision lenticule extraction for myopia. Clin Ophthalmol. 2018; 12:1741-1755.

5. Khalifa MA, Ghoneim AM, Shaheen MS, et al. Vector analysis of astigmatic changes after smallincision lenticule extraction and wavefront-guided laser in situ keratomileusis. J Cataract Refract Surg. 2017; 43(6):819-824.

6. Chan TC, Ng AL, Cheng GP, et al. Vector analysis of astigmatic correction after small-incision lenticule extraction and femtosecond-assisted LASIK for low to moderate myopic astigmatism. $\mathrm{Br} J$ Ophthalmol. 2016; 100(4):553-559.

7. Shen EP, Chen WL, Hu FR. Manual limbal markings versus iris-registration software for correction of myopic astigmatism by laser in situ keratomileusis. J Cataract Refract Surg. 2010; 36(3):431-436.

8. Alpins N. Astigmatism analysis by the Alpins method. J Cataract Refract Surg. 2001; 27(1):31-49.

9. Chan TCY, Wang Y, Ng ALK, et al. Vector analysis of high ( $\geq 3$ diopters) astigmatism correction using small-incision lenticule extraction and laser in situ keratomileusis. Journal of Cataract \& Refractive Surgery. 2018; 44(7):802-810.

10. Reinstein DZ, Archer TJ, Randleman JB. JRS standard for reporting astigmatism outcomes of refractive surgery. J Refract Surg. 2014; 30(10):654-659.

11. Gauvin M, Wallerstein A. AstigMATIC: an automatic tool for standard astigmatism vector analysis. BMC Ophthalmol. 2018; 18(1):255.

12. Liu M, Chen Y, Wang D, et al. Clinical Outcomes After SMILE and Femtosecond Laser-Assisted LASIK for Myopia and Myopic Astigmatism: A Prospective Randomized Comparative Study. Cornea. 2016; 35(2):210-216.

13. Han T, Xu Y, Han X, et al. Three-year outcomes of small incision lenticule extraction (SMILE) and femtosecond laser-assisted laser in situ keratomileusis (FS-LASIK) for myopia and myopic astigmatism. Br J Ophthalmol. 2019; 103(4):565-568.

14. Agca A, Demirok A, Cankaya Kl, et al. Comparison of visual acuity and higher-order aberrations after femtosecond lenticule extraction and small-incision lenticule extraction. Cont Lens Anterior Eye. 2014, 37(4):292-296. 
15. Zhang J, Wang Y, Chen X. Comparison of Moderate- to High-Astigmatism Corrections Using WaveFront-Guided Laser in Situ Keratomileusis and Small-Incision Lenticule Extraction. Cornea. 2016; 35(4):523-530.

16. Zhao X, Zhang L, Ma J, et al. Comparison of Wavefront-Guided Femtosecond LASIK and Optimized SMILE for Correction of Moderate-to-High Astigmatism. J Refract Surg. 2021; 37(3):166-173.

17. Ganesh S, Brar S, Pawar A. Results of Intraoperative Manual Cyclotorsion Compensation for Myopic Astigmatism in Patients Undergoing Small Incision Lenticule Extraction (SMILE). J Refract Surg. 2017; 33(8):506-512.

18. Pedersen IB, Ivarsen A, Hjortdal J. Changes in Astigmatism, Densitometry, and Aberrations After SMILE for Low to High Myopic Astigmatism: A 12-Month Prospective Study. J Refract Surg. 2017; 33(1):11-17.

19. Kanellopoulos AJ, Asimellis G: Longitudinal postoperative lasik epithelial thickness profile changes in correlation with degree of myopia correction. J Refract Surg 2014, 30(3):166-171.

20. Zhang J, Wang Y, Wu W, et al. Vector analysis of low to moderate astigmatism with small incision lenticule extraction (SMILE): results of a 1-year follow-up. BMC Ophthalmol. 2015; 15:8.

21. Alpins NA. Vector analysis of astigmatism changes by flattening, steepening, and torque. J Cataract Refract Surg. 1997; 23(10):1503-1514.

22. Kanellopoulos AJ. Topography-Guided LASIK Versus Small Incision Lenticule Extraction (SMILE) for Myopia and Myopic Astigmatism: A Randomized, Prospective, Contralateral Eye Study. J Refract Surg. 2017; 33(5):306-312.

23. Woo YJ, Lee H, Kim HS, et al. Comparison of 3 marking techniques in preoperative assessment of toric intraocular lenses using a wavefront aberrometer. J Cataract Refract Surg. 2015; 41(6):12321240.

24. Prickett AL, Bui K, Hallak J, et al. Cyclotorsional and non-cyclotorsional components of eye rotation observed from sitting to supine position. Br J Ophthalmol. 2015; 99(1):49-53.

25. Jun I, Kang DSY, Reinstein DZ, et al. Clinical Outcomes of SMILE With a Triple Centration Technique and Corneal Wavefront-Guided Transepithelial PRK in High Astigmatism. J Refract Surg. 2018; 34(3):156-163.

26. Ivarsen A, Asp S, Hjortdal J. Safety and complications of more than 1500 small-incision lenticule extraction procedures. Ophthalmology. 2014;121(4):822-828.

27. Varma R, Torres M, McKean-Cowdin R, et al. Chinese American Eye Study G: Prevalence and Risk Factors for Refractive Error in Adult Chinese Americans: The Chinese American Eye Study. Am J Ophthalmol. 2017; 175:201-212.

28. Pesudovs K. Autorefraction as an outcome measure of laser in situ keratomileusis. J Cataract Refract Surg. 2004; 30(9):1921-1928.

\section{Figures}




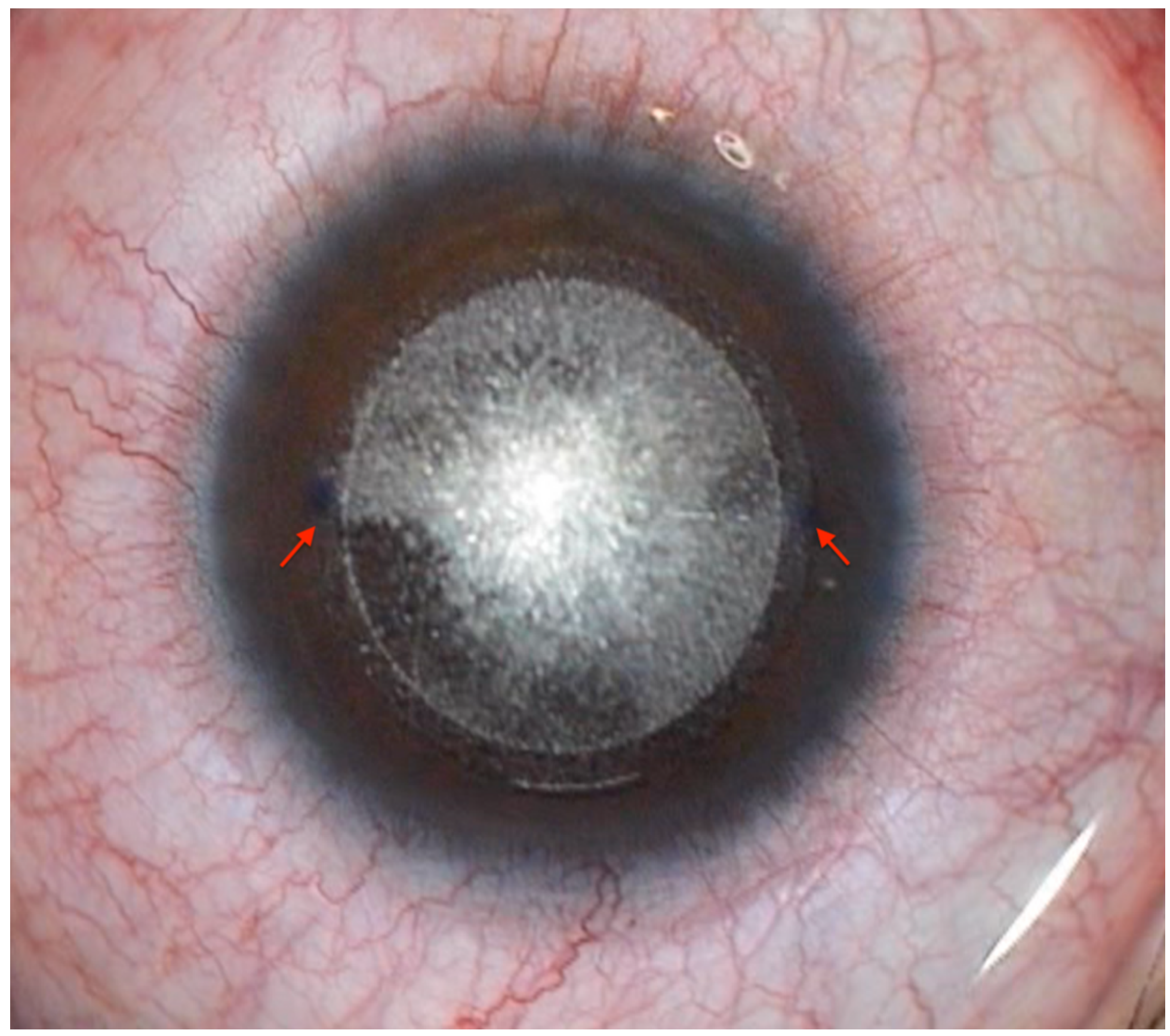

Figure 1

Limbal marking at the $0^{\circ}$ to $180^{\circ}$ axis in front of the slit-lamp preoperatively 


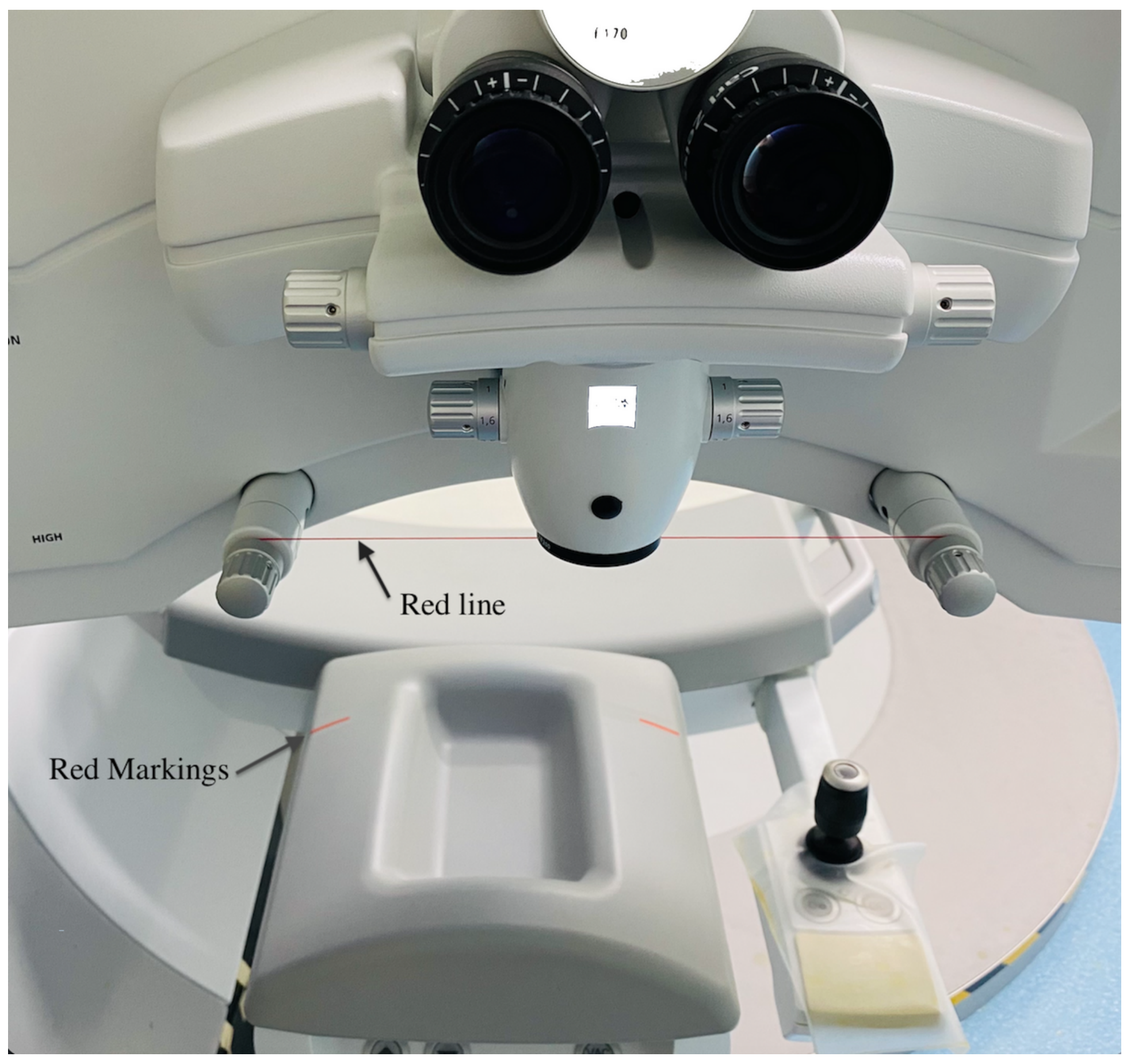

Figure 2

Red markings on the operating bed: The surgical assistant makes the edge of the patient's earlobes correspond to the red markings; Red line: the surgeon adjusts the patient's inner and outer canthal angle parallel to the red line 

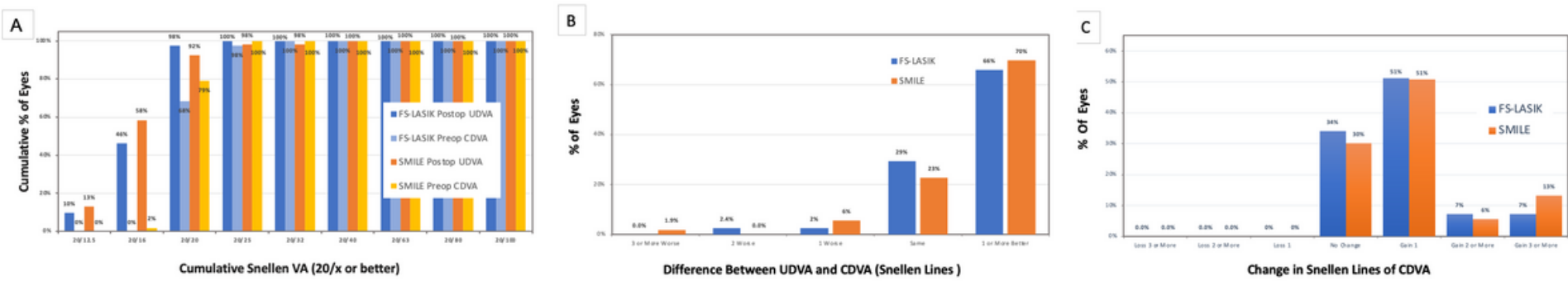

\section{Figure 3}

Efficacy and safety for FS-LASIK and SMILE at 12-month. The postop UDVA compared with the preop CDVA (A); postop UDVA vs preop CDVA (B); Change in lines of postop CDVA (C). UDVA= Uncorrected Distance Visual Acuity, CDVA=Corrected Distance Visual Acuity

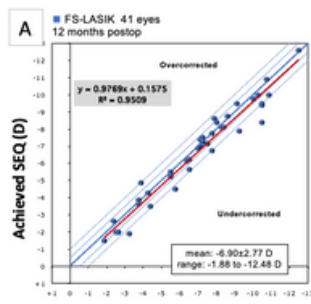

Attempted SEQ (D)

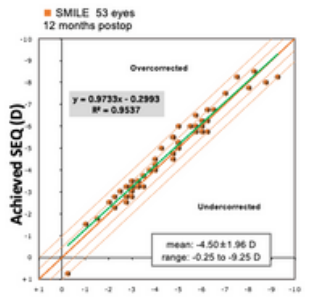

Attempted SEQ (D)

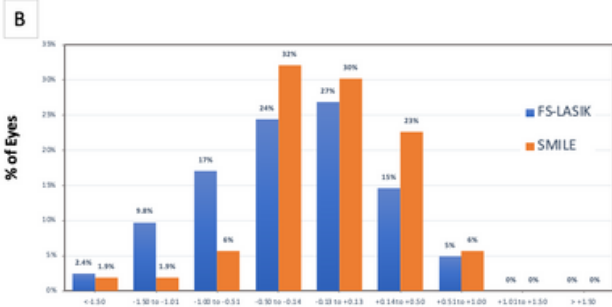

Accuracy of SEQ to Intended Target (D)

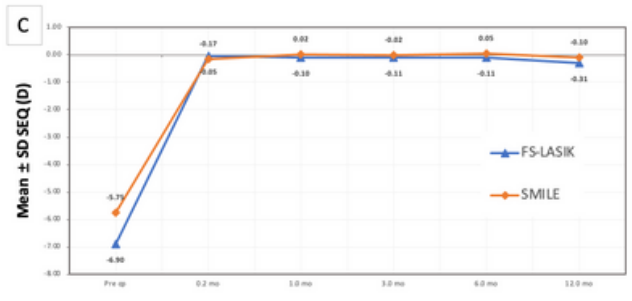

Time After Surgery (months)

Figure 4

The predictability of spherical equivalent refraction for FS-LASIK and SMILE at 12-month. The attempted vs achieved spherical equivalent refraction (A); Spherical Equivalent Refraction Accuracy (B); Spherical Equivalent Refraction Stability (C)
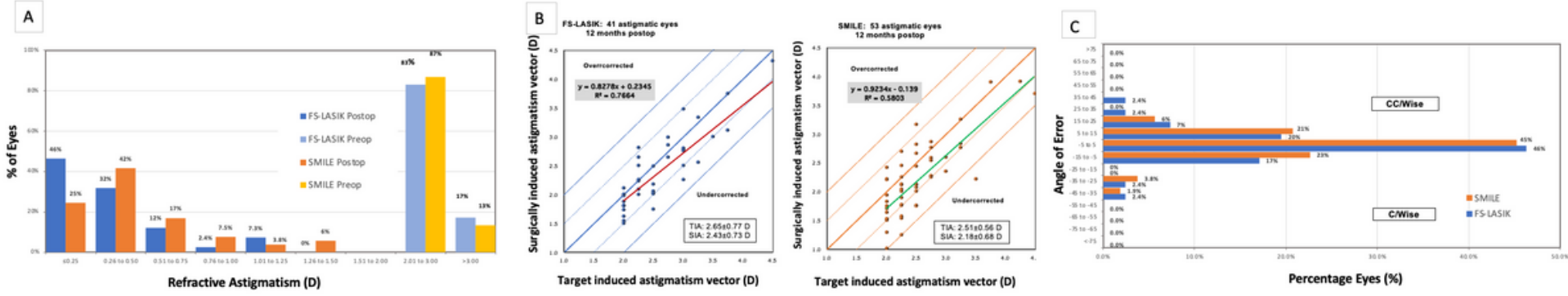

Figure 5

The predictability of cylindrical refraction for FS-LASIK and SMILE at 12-month. Refractive Astigmatism of postop vs preop (A); TIA vs SIA (B); Refractive Astigmatism Angle of Error (C) (SIA = surgically induced astigmatism; TIA = target-induced astigmatism) 

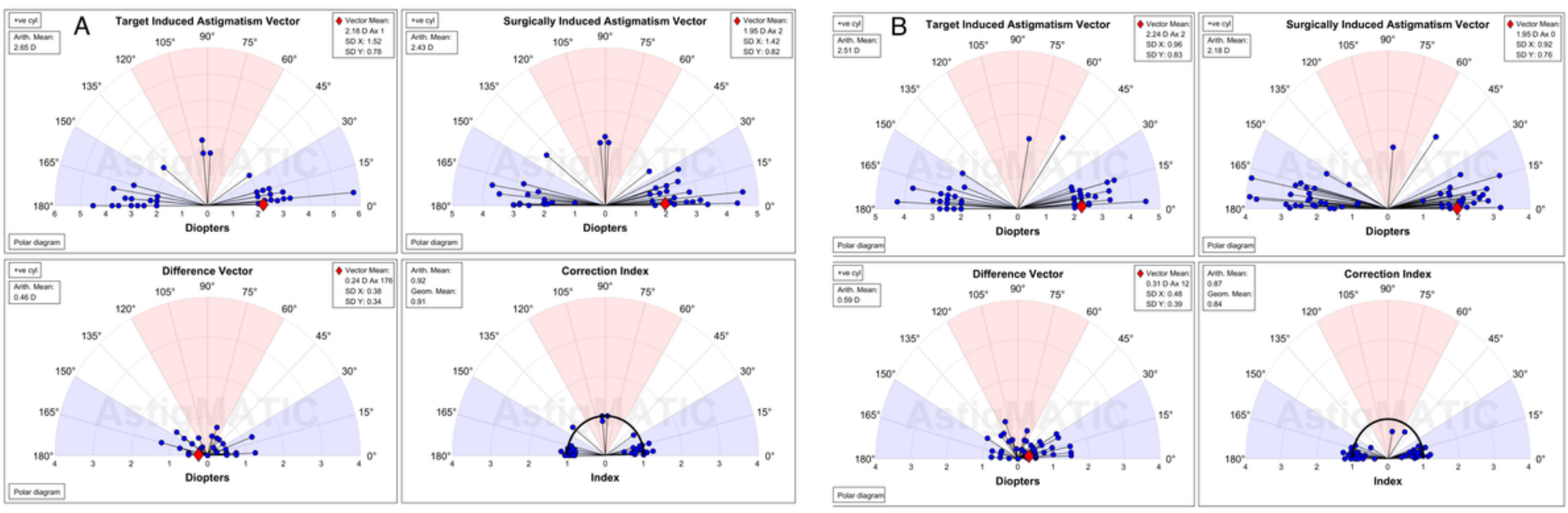

Figure 6

Single angle polar plots of TIA, SIA, difference vector, and correction index at 12 months after FS-LASIK (A) and SMILE (B) (SIA = surgically induced astigmatism; TIA = target-induced astigmatism) 